\title{
Application of biodegradable Aloe vera gel for extending the shelf-life of tomato
}

\author{
Kanmani, V. Meenakshi and G. Sashidevi
}

\begin{abstract}
The search for biodegradable, safe, healthy and environmental friendly treatments for increasing storage period of fruits has lead to use of edible, biodegradable films or coatings. Biopreservations is a novel food preservation method defined for extension of shelf-life and enhanced safety of foods by the use of natural or controlled microbiota and/or antimicrobial compounds. Aloe vera gel is applied to fruits as an edible coating which has been widely used for most fruits and vegetables. The aim of this work was to analyze the effects of biodegradable Aloe vera gel (aloe alone,1:1,1:2) along with $1 \%$ pectin or CMC coating on tomato stored at room temperature. Matured leaves of Aloe vera plants were harvested and washed and gel was extracted. The viscosity of Aloe vera gel and its coating efficiency was improved by using $1 \%$ thickening agent such as $\mathrm{CMC}$ and pectin. Aloe vera gel (Aloe vera juice mixed with $1 \% \mathrm{CMC} / 1 \%$ pectin) was taken in three different concentrations i.e., alone alone, $1: 1(200 \mathrm{ml}$ of Aloe vera gel: $200 \mathrm{ml}$ of water), 1:2 (133.3 $\mathrm{ml}$ of Aloe vera gel: $266.6 \mathrm{ml}$ of water). The matured tomatoes ( $80 \%$ ) were dipped in the coating solutions at room temperature for $10 \mathrm{~min}$. At regular intervals, the fruits were rotated to increase the coating efficiency. They were allowed to drain for $2 \mathrm{~min}$ and then dried at room temperature under fan, to increase drying rate. The treated samples were packed in 100 gauge polyethylene bags with $1 \%$ vent and another set of samples were kept without packing. The control and treated samples were stored at room temperature. The results revealed aloe gel coating to be very beneficial in reducing the weight loss, minimizing changes in physico-chemical parameters (titrable acidity, total soluble solids, lycopene) of the fresh fruits. The overall result showed that the Aloe vera gel coatings along with CMC and pectin is effective in extending the shelf-life of tomato fruits when compared to untreated tomato in the following order: Aloe +pectin (without packing) $>$ Aloe $+\mathrm{CMC}$ (without packing ) > Control. In respect of combined effects, tomato treated with Aloe vera $+1 \%$ pectin(without packing) showed the longest shelf-life ( 20 days), followed by Aloe vera $+1 \%$ CMC (without packing) whereas the shortest shelf-life ( 8 days) was recorded in control condition.This work evaluates the use of Aloe vera as biopreservative, which is an economical and eco-friendly.
\end{abstract}

Key Words : C M C ( Carboxy methyl cellulose), Pectin

How to cite this article : Kanmani, Meenakshi, V. and Sashidevi, G. (2017). Application of biodegradable Aloe vera gel for extending the shelf-life of tomato. Food Sci. Res. J., 8(2): 132-137, DOI : 10.15740/HAS/FSRJ/8.2/132-137. 